\title{
Characteristics of Phosphate-Solubilizing Bacteria Isolated from Acid Soil of Cikopomayak, West Java, Indonesia
}

\author{
HAPPY WIDIASTUTI
}

\author{
Indonesian Biotechnology Research Institute for Estate Crops, Jalan Taman Kencana No. 1, Bogor 16151, Indonesia \\ Phone: +62-251-8324048, Fax:+62-251-8328516,E-mail: happywidiastuti@yahoo.com
}

\begin{abstract}
Phosphate-solubilizing bacteria were isolated from acid soil from Cikopomayak. Microbiological assay showed that the bacteria were Gram negative, rod-shaped, and lacked red pigment on Pikovskaya medium. Isolate A synthesized red pigment on nutrient agar medium, while isolate B formed slightly red pigment on nutrient agar medium as well as on Voges Proskauer medium. The ratio of clearing zone to colony for isolate A and B were approximately 2.1 and 1.9 , respectively. Biochemical assays showed that both isolates A and B utilized glucose, lactose, sucrose, mannitol, glycerol, mannose, urea, indole, and produced catalase, but neither produced amylase and oxidase. The bacteria are identified as Serratia marcescens. The ability to solubilize phosphate decreased or was even lost when subcultured on nutrient agar medium. However, the ability to solubilize phosphate was recovered gradually with the addition of phosphate. The results presented here suggest that the ability to solubilize phosphate and to synthesize the red pigment may be inversely correlated.
\end{abstract}

Key words: acid soil, Serratia marcescens, phosphate-solubilizing bacteria, medium, pigment

The bacterium Serratia sp. has been isolated from soil, water, plants, air, as well as from the gut of various insects and vertebrates. It is also found in the intestine of humans. Species of Serratia are Gram negative, rod-shaped, ureasolubilizing and non-spore-forming (Bhadra et al. 2005). These bacteria produce red pigment prodigiosin, a member of the prodiginines, which is produced by some Serratia species, actinomycetes and a few other bacteria. Prodigiosins have no defined role in the physiology of producing strains, but have been reported to have antifungal, anti bacterial and anti protozoal/anti malarial activities.

The ability of some Serratia marcescens strains to colonize a wide range of ecological niches has been linked to the production of a spectrum of extracellular products including chitinase, protease, lipase, nuclease, anti-bacterial agents, surfactants and wetting agents (Matsuyama et al. 1995). Gyaneshwar et al. (2002) showed that S. marcescens was an endophytic bacteria of rice that could increase the root length and dry weight. The report of DBT 2003-2004 showed that $S$. marcescens isolated from the tea rhizosphere was antagonistic to all plant pathogenic fungi. The assay showed that the bacteria increased plant growth, plant height, the number of shoots, the number of leaves per shoot and the phenolic chlorophyll content. In addition, a Serratia species, S. phosphaticum, was reported to be able to solubilize Pi as a result of the production of organic acids such as gluconic and 2-keto-gluconic acids (Tilak et al. 2005). In humans, selected Serratia strains cause nosocomial infection that is clinically problematic because of multidrug resistance in some species (Harris et al. 2004).

\section{MATERIALS AND METHODS}

Bacteria were isolated using Pikovskaya medium at room temperature. Morphological characterization was observed by growing the bacteria on nutrient agar (NA). Fresh cell preparations of the bacteria were microscopically examined using a light microscope at $1000 \mathrm{X}$ magnification. Biochemical characterizations consisted of catalase assay, testing for growth and acid production on glucose and lactose, sucrose, mannitol, glycerol, mannose and Methyl Red-Voges Proskauer (MR-VP). In addition, testing was done for the production of amylase, urea, indole, oxidase (catalyzed oxidation of cytochrome to $\mathrm{H}_{2} \mathrm{O}_{2}$ ) and citric acid. Selected physiological characteristics were assessed, such as growth and pigment synthesis on NA, Pikosvkaya and VP media. The bacteria were maintained on NA medium.

As the bacteria were subcultured in NA, the ability to solubized Pi was loss. In effort to recover the lost ability to solubilize $\mathrm{Pi}$, the bacteria were grown on Pikovskaya medium containing various levels of Pi. Three levels of Pi were examined i. e. as in Pikovskaya medium (1.6 $\left.\mathrm{mg} \mathrm{Pi} \mathrm{ml}^{-1}\right) ; 50 \%$ of the Pi level in Pikovskaya medium ( $\left.0.8 \mathrm{mg} \mathrm{Pi} \mathrm{ml}^{-1}\right) ; 150 \%$ of Pi level in Pikovskaya medium (2.4 $\mathrm{mg} \mathrm{Pi} \mathrm{ml}^{-1}$ ).

\section{RESULTS}

The bacteria were isolated using dilution and surface plating methods on Pikovskaya media. Two isolates of phosphate-solubilizing bacteria were obtained. Table 1 shows that both isolate A and B produce catalase and use glucose, sucrose, mannitol, glycerol and mannose as $\mathrm{C}$ sources. There were no significant differences between isolate A and B except for the color of the colonies. In NA medium, isolate A initially produced red pigment that was much darker compared to that of isolate B. However, there were color differences for the colonies on NA and on Pikovskaya media (Fig 1). On Pikovskaya medium, colonies were, white while on NA they were red. Another difference was the ability to solubilize Pi. The ratio of diameter of the clearing zone to that of the colony for isolate A was 2.1 and for isolate B was 1.9.

The bacteria were subcultured on NA media to maintain them. After several subculture steps, the bacteria were not able to solubilize Pi on Pikovskaya medium but still produced red pigment (Fig 2). Subculturing of these two isolates in 
Pikovskaya agar medium resulted in the loss of ability to produce red pigment. In this medium the isolates still could not solubilize Pi. Recovery of the Pi solubilizing ability of both isolates was accomplished by gradually increasing the dose of Pi in Pikovskaya medium. There were differences in the dose of Pi required for Pi solubilization activity between two isolates. Isolate B initially solubilized Pi in Pikovskaya medium at a $50 \%$ Pi concentration $(10 \mathrm{mM})$, but recovery of Pi solubilizing ability for isolate A was induced with the addition of $25 \% \mathrm{Pi} \mathrm{(5} \mathrm{mM)} \mathrm{(Fig} \mathrm{3).}$

Table 1 Morphological and physiological traits of two isolates of Serratia marcescens

\begin{tabular}{lcc}
\hline Characteristics & Isolate A & Isolate B \\
\hline Morphology of colony shape & slimy & slimy \\
Gram & negative & negative \\
Cell shape & rod & rod \\
Physiological traits: & + & \\
$\quad$ Catalase activity & + & + \\
$\quad$ Glucose & - & + \\
Lactose & + & + \\
Sucrose & + & + \\
Mannitol & + & + \\
Glycerol & + & + \\
Mannose & - & - \\
Urea & - & - \\
Indole & - & - \\
Oxidase & - & - \\
Amylum (amylase activity) & + & + \\
Citrate & - & + \\
Methyl red & + & \\
Voges proskauer & & - \\
Colony color: & red & slightly red \\
Nutrient agar & - & red \\
Pikovskaya & red & \\
Voges proskauer & &
\end{tabular}
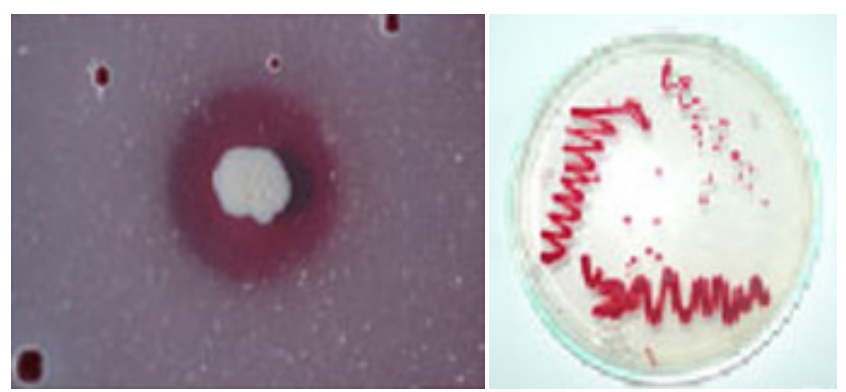

Fig 1 Color difference produced by phosphate-solubilizing bacteria isolated from Cikopomayak acid soil. On Pikovskaya medium the bacteria could not synthesize pigment (left) while on NA the bacteria synthesized red pigment (right).

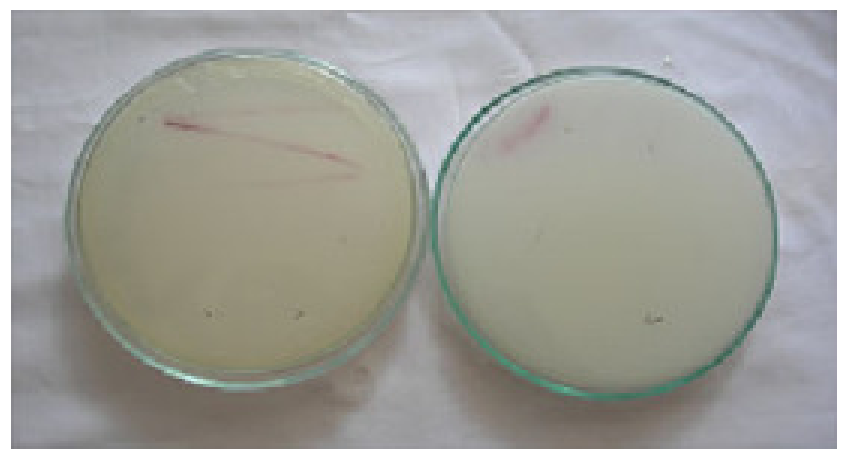

Fig 2 Inability of isolate A and B to solubilize Pi and synthesize pigment in Pikovskaya + NA (left) and Pikovskaya (right) after subculturing in NA.

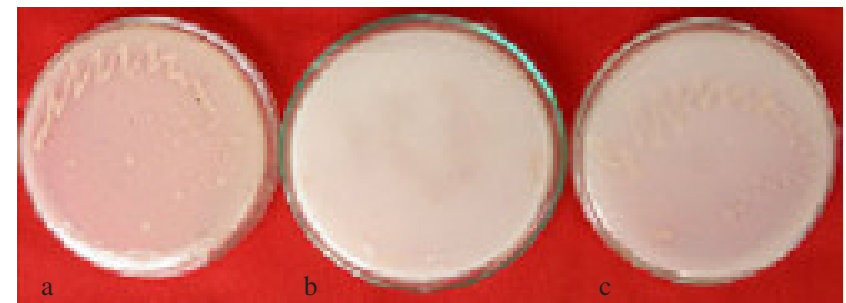

Fig 3 Regaining of Pi-solubilizing ability of isolate A induced by gradual addition of $\mathrm{Pi}$ in Pikovskaya medium. (a) initial $\mathrm{Pi}$ concentration (50\%); (b) defined Pikovskaya medium (100\%); (c) $25 \%$ Pi addition.

\section{DISCUSSION}

Based on selected characteristics, isolate A and B were identified as $S$. marcescens. Table 1 showed that those two isolates of phosphate-solubilizing bacteria did not utilize urea. This characteristic was in line with that of $S$. marcescens as reported by Bhadra et al. (2005). Bhadra et al. (2005) also reported that another Serratia species, S. ureilytica did utilize urea. The results therefore showed that there were variations between species in Serratia. In addition, the ability to solubilize Pi between isolates A and B was slightly different. Based on the ratio of the diameter of the clearing zone to colony, the ability of isolate A in solubilizing Pi was higher than that of isolate B.

The interesting characteristics of these bacteria were the differences in pigment production on selected media (Table 1). It was assumed that the differences in the ability to synthesize pigment was affected by medium composition. Pikovskaya medium is a simple, defined medium, while NA is a complex medium. The content of both organic and inorganic components in Pikovskaya is precisely measured, while in NA the content of the medium substituents is uncertain because NA media contains beef and yeast extracts. On Pikovskaya medium, the bacteria must synthesize all of their organic compounds from glucose. However, on NA, nutrients required to form organic compounds for cell growth are available. The same is true for VP medium, which is also a complex medium. On this medium the isolates formed pigment (Table 1). The organic compounds present in VP medium are glucose and peptone. Although glucose is the macronutrient utilized to form gluconic acid, which is needed to solubilize $\mathrm{Pi}$, it can also be used to form pigment. It might cause the differences in physiology especially in catabolism to produce energy, hence, the ability to synthesize pigment.

The differences in pigment synthesis on Pikovskaya medium and NA might also be caused by the difference in the $\mathrm{N}$ source of the media. Williams (1973) showed that the precursor of prodigiosin synthesis were serine, alanine, methionine and proline. Pikovskaya medium (pigment -) contained $\mathrm{N}$ in inorganic form i.e. ammonium sulphate while in NA (pigment + ), beef extract and peptone were the source of N. Peptone is also the $\mathrm{N}_{2}$ source found in VP medium.

The red pigment prodigiosin results from secondary metabolism. Fineran et al. (2005) reported that prodigiosin synthesis involved quorum sensing mediated by $\mathrm{N}$-acyl homoserine lactone (N-AHL). They showed that there was a transcriptional activator PigT. PigT activated transcription of biosynthestic pig A-O operon in the absence of gluconic 
acid. The addition of gluconic acid inhibited pig A-O transcription. It was also reported that $\mathrm{P}$ solubilization by phosphate-solubilizing Gram-negative bacteria was caused by the presence of 2-keto-gluconic acid (Rodriquez et al. 2001). It is possible that the lack of pigment formation in Pikovskaya medium is caused by gluconic acid synthesis by phosphate-solubilizing bacteria. Rodriquez et al. (2001) showed that the high Pi solubilizing ability of Gram negative bacteria was caused by gluconic acid and 2-keto-gluconic acid synthesized in the periplasmic space from glucose oxidation through quinoprotein-glucose-dehydrogenase (EC1.1.5.2). This result suggested that the synthesis of pigment in $S$. marcescens was inhibited by phosphatesolubilizing activity. However, Slater et al. (2003) showed that phosphate availability regulates biosynthesis of prodigiosin in Serratia via both quorum-sensing-dependent and independent pathways. They showed that in Serratia 39006 Pi limitation was a cause of prodigiosin operon upregulation.

Gluconic acid that was needed in solubilizing phosphate was synthesized from glucose in oxidation reactions (Rodriguez et al. 2001). As a result, in reducing conditions the bacteria were unable to solubilize Pi. It was reported that by reducing oxygen pressure, $S$. marcescens did not form pigment, while under the microaerobic conditions, the pigment was formed. In this situation the oxygen is used to produce $\mathrm{CO}_{2}$. It seemed that the growth of those two isolates were similar in inducing both solubilizing Pi ability and pigment synthesis whether they were incubated in Pikovskaya or NA.

In our experiments, after several subcultures, the ability to solubilize Pi on Pikovskaya medium was lost when subcultured on NA, but the bacteria still produced pigment. This supported the hypothesis that pigment synthesis inhibited Pi solubilization. The lack of ability to synthesize red pigment in the presence of Pi (initially) caused the suspicion that in pigment synthesis Pi nutrient must be available. On the other hand, the inability of the isolate to form clear zone in NA was caused by the sufficient availability of $\mathrm{Pi}$ and the inhibition of gluconic acid synthesis needed in Pi solubilization. The ability to form red pigment in NA medium was made possible by the presence of gluconic acid that inhibited the pig-T-activator transcript as reported by Fineran et al. (2005). The results suggested that in prodigiosin synthesis, Pi should be available. In addition, it was suspected that the bacteria were able to solubilize Pi only when Pi was not available.

Bacterial Pi solubilization might be caused by the synthesis of hydroxy acids such as oxalic acid that may chelate with $\mathrm{Ca}^{2+}$ and $\mathrm{Fe}^{2+}$. If this is the mechanism of $\mathrm{Pi}$ solubilization, then it raises some questions: is there any correlation between oxalic acid and gluconic acid? Is gluconic acid the oxalic acid precursor? Does prodigiosin synthesis suppress the synthesis of organic acid? Is there a common precursor for organic acid synthesis and prodigiosin synthesis?

The observation of Pi-solubilizing-ability of the bacteria at various levels of Pi revealed that the ability of isolate B to solubilize Pi was regained on medium containing 50\% Pi concentration, while on this medium isolate A could still not solubilize Pi. On Pikovskaya medium containing 150\% Pi concentration, neither isolate A nor B solubilized Pi. The ability of isolate A was recovered when grown on medium with only $25 \% \mathrm{Pi}$ level of the Pikovskaya medium. These results indicate that the ability of isolates $\mathrm{A}$ and $\mathrm{B}$ in solubilizing $\mathrm{Pi}$ are different. The recovery of Pi-solubilizingability of isolate A was slower compared to that of isolate B. Different recovery processes between isolates A and B indicated a correlation between the ability to solubilize $\mathrm{Pi}$ and pigment synthesis even though at the beginning of isolation the bacteria showed both the ability to solubilize Pi and synthesize pigment. It was suspected that soil contained compounds that could induce these two processes. Synthetic media like NA medium and Pikovskaya lacked these compounds, resulting in physiological changes in the bacteria.

These results showed that available Pi inhibited the ability of bacteria to solubilize Pi. The ability of isolate A and B to solubilize Pi changes according to the media composition, especially the presence of $\mathrm{C}$ and $\mathrm{N}$ compounds and the availability of $\mathrm{Pi}$. The recovery of solubilization activity between isolates A and B was different. Phosphatesolubilizing activity of isolate $\mathrm{A}$ was higher compared to that of isolate B. It was in line with the recovery of the ability to solubilize Pi. Isolate B had less ability than isolate A in solubilizing $\mathrm{Pi}$ as shown by ratio of clearing zone and colony that explains why it needed more Pi compared to isolate A.

Considering these results, could the ability of bacteria to solubilize Pi be increased by inhibiting pigment synthesis? Likewise, could the formation of red pigment be induced by increasing the availability of free P? It was suggested that $\mathrm{Pi}$ - solubilization and pigment synthesis could be controlled by controlling the addition of Pi. These results indicated that in nature, in an environment with low availability of $\mathrm{Pi}$, the bacteria synthesize gluconic acid that can lead to solubilization of $\mathrm{Pi}$, while in an environment with sufficient Pi the bacteria synthesize pigment.

This paper showed that there were physiological changes in the bacteria. The changes could easily be observed through the appearance of pigment and the formation of clearing zone. This paper reports the characterization of $S$. marcescens isolated from acid soil of Cikopomayak. Other characteristics such as the interactions of the bacteria with plants need to be studied further.

\section{REFERENCES}

Bhadra B, Roy P, Chakraborty R. 2005. Serratia ureilytica sp. nov., a novel urea-utilizing species. Int J Syst Ecol Microbiol 55:21552158.

Fineran PC, Everson L, Slater H, Salmond GPC. 2005. A GntR family transcriptional regulator (PigT) controls gluconate-mediated repression and defines a new, independent pathway for regulation of the tripyrrole antibiotic, prodigiosin, in Serratia. Microbiology $151: 3833-3845$

Gyaneshwar P, Janes EK, Mathan N, Reddy PM, Reinhold-Hweh B, Ladha JK. 2002. Endophytic colonization of rice by a diazothropic strain of Serratia marcescens. J Bacteriol 183:26342643.

Harris AKP, Williamson NR, Slater H, Cox A, Abbasi S, Foulds I, Simonsen HT, Leeper FJ, Salmond GPC. 2004. The Serratia gene cluster encoding biosynthesis of the red antibiotic, prodigiosin, shows species and strain-dependent genome context variation. Microbiology 150:3547-3560. 
Matsuyama T, Bhesin A, Harshey RM. 1995. Mutational analysis of flegelin-independent surface spreading of Serratia marcescens 274 on a low agar medium. J Bacteriol 177:987-991.

Rodriquez H, Gonzalez T, Selma G. 2001. Expression of a mineral phosphate solubilizing gene from Erwinia herbicida in two rhizo bacteroid strain. J Biotechnol 84:155-161.

Slater H, Crow M, Everson L, Salmond GP. 2003. Phosphate availability regulates biosynthesis of two antibiotics, prodigiosin and carbapenem, in Serratia via both quorum-sensing-dependent and-independent pathways. Mol Microbiol 47:303-320.

Tilak KVBR, Ranganayaki N, Pal KK, De R, Saxena AK, Nautiyal CS, Mittae S, Tripathi AK, Jahri BN. 2005. Diversity of plant growth soil health supporting bacteria. Curr Sci 89:134-150.

Williams RP. 1973. Biosyinthesis of prodigiosin, a secondary metabolite of Serratia marcescens. Appl Microbiol 25:396-402. 